\title{
Stochastic High Order Basis Functions for Volume Integral Equation with Surface Roughness
}

\author{
Tarek Moselhy and Luca Daniel \\ Research Laboratory of Electronics, Massachusetts Institute of Technology \\ Email: \{tmoselhy,luca\}@mit.edu
}

\begin{abstract}
In this paper we present stochastic high order basis functions suitable for the volumetric discretization of interconnect structures. The proposed basis functions are wideband, and can easily be integrated within a stochastic magneto-quasistatic (MQS) or a fullwave Mixed Potential Integral Equation (MPIE) solver, rendering the stochastic formulation computationally efficient. In addition, our high order basis functions facilitate the calculation of a correction term, improving the accuracy of the impedance ensemble average.
\end{abstract}

\section{INTRODUCTION}

Stochastic electromagnetic simulation has become an appealing alternative to Monte Carlo simulations, in order to effectively account for on-chip process variations and off-chip surface roughness effects [1], [2]. In a companion paper submitted to this conference [3] we have developed a volumetric magneto-quasistatic (MQS) formulation accounting for surface roughness effects. In such approach, since we are mainly interested in the average current distribution, and since the surface roughness can me modeled by an ergodic process, we are able to discretize complicated interconnects into relatively long wire segments. However, a very large number of thin cross sectional volumetric filaments may be required to accurately describe the current within each segment and accurately account for roughness effects, since the correlation length of the surface roughness can be very small relative to the cross section. High order basis functions have been proposed and developed in [4], [5] as an efficient way to reduce the number of cross sectional basis functions in deterministic solvers. In this paper we make use of a similar wideband basis function generation technique, and extend it further to account for stochastic variations.

\section{BACKGROUND}

\section{A. Formulation and Surface Model}

In this paper, we are primarily interested in the equation governing the relation between the current density $J$ inside the volume of the wires, and the scalar potential $\phi$,

$$
\rho J(r)+j \omega \int_{V} G\left(r, r^{\prime}\right) J\left(r^{\prime}\right) d V=-\nabla \phi, \quad \nabla \cdot J=0,
$$

where $G\left(r, r^{\prime}\right)=\mu /\left(4 \pi\left|r-r^{\prime}\right|\right)$ is the free space Green's function, $r$ and $r^{\prime}$ are observation and source points, respectively; $\rho$ is the resistivity of the conductor, and $\omega$ is the frequency. The volume of the conductors $V$ is bounded by rough surfaces, i.e. the domain of integration is described by a stochastic Gaussian process of the form:

$$
\begin{gathered}
P_{2}\left(X_{2}, Y_{2}, Z_{2}, H_{2}\right)=\frac{\exp \left(-0.5 H_{2}^{T} \Sigma_{2}^{-1} H_{2}\right)}{2 \pi \sqrt{\left|\Sigma_{2}\right|}} \\
X_{2}=\left[\begin{array}{c}
x_{i} \\
x_{j}
\end{array}\right], \quad Y_{2}=\left[\begin{array}{c}
y_{i} \\
y_{j}
\end{array}\right], \quad Z_{2}=\left[\begin{array}{c}
z_{i} \\
z_{j}
\end{array}\right], \quad H_{2}=\left[\begin{array}{c}
h_{i} \\
h_{j}
\end{array}\right], \quad \Sigma_{2}=\sigma^{2}\left(\begin{array}{cc}
1 & C \\
C & 1
\end{array}\right), \quad C=\exp \left(-\frac{\left\|r_{i}-r_{j}\right\|^{2}}{L^{2}}\right)
\end{gathered}
$$

where $h_{i}$ and $h_{j}$ are the surface heights at locations $r_{i}=\left(x_{i}, y_{i}, z_{i}\right)$ and $r_{j}=\left(x_{j}, y_{j}, z_{j}\right)$, respectively. $\sigma$ and $L$ are the standard deviation and the correlation length of the rough surface, respectively.

A standard procedure for solving (1) involves discretizing the current density $J$ using some basis functions and a Galerkin technique, to obtain the resistance and partial inductance matrices $R$ and $L$ [6]. The current conservation constraint in (1) can be imposed using a mesh analysis matrix $\mathrm{M}$ and mostly zero KVL vector $\Delta \Phi_{m}$, to obtain as in [6] a linear system $M(R+j \omega L) M^{T} I_{m}=\Delta \Phi_{m}$, that can be solved for the mesh currents $I_{m}$. 


\section{B. Wideband Proximity Templates Basis Functions}

High order basis functions discretizing (1) can be constructed using numerical simulation to generate "typical" cross section current distributions as in [4]. According to such procedure, one first subdivides complicated interconnects into wire segments. For each type of segment cross-section, one calculates numerically the cross-sectional current distributions resulting from different locations of a nearby wire carrying the return current. The collections of all such current distributions represents a basis function subspace that can span and capture skin and proximity effects. Such calculations can be carried out very efficiently since they involve solving (1) on a geometry including only two wires, that can therefore be discretized using for instance simple thin filaments (piecewise constant basis functions). Finally, for wideband basis functions that can generate well-conditioned systems we can, as in [5], generate cross-section current distributions at a large number of frequency points and proximity configurations, assemble them as columns of a matrix, use singular value decomposition to obtain the principal orthogonal vectors, and optimize them to guarantee a physical non-zero current sum.

\section{STOCHASTIC HIGH ORDER BASIS FUNCTIONS}

In this paper we use the wideband proximity templates basis functions generation procedure described in [4], [5], and summarized in II-B. However, in our case, the cross sectional area of the conductor is defined by rough surfaces, and is therefore not deterministic. When generating numerically the high order basis function as in II-B, our discretization strategy relies on pre-defining the number of thin filaments per cross-section, such that the largest cross-sectional filament size is smaller than a preset threshold for any possible surface realization within $3 \sigma$ from the mean smooth surface. We further use non-uniform filament sizes to capture skin and proximity effects near the conductor boundaries more efficiently. To store each numerically generated cross-sectional current density we use a vector $I$ of length $K$, equal to the number of thin filaments discretizing the wire cross-section during the generation process. The high order basis functions, representing the current density in a given wire segment, is therefore

$$
B(r)=\sum_{k=1}^{K} \frac{I[k]}{A_{k}} v_{k},
$$

where $A_{k}$ is the random cross-sectional area of the $k^{t h}$ thin filament $V_{k}$. Vector $v_{k}=1$ if $r \in V_{k}$, otherwise $v_{k}=0$.

The ensemble average (denoted by the overline) for the elements of the matrices $L$ and $R$ generated by our stochastic high order basis functions are given by:

$$
\begin{aligned}
\bar{L}_{i j} & =\iint_{X_{2}} \iint_{Y_{2}} \iint_{\substack{H_{2} \\
z_{i}=f\left(h_{i}\right), z_{j}=f\left(h_{j}\right)}} P_{2}\left(X_{2}, Y_{2}, Z_{2}, H_{2}\right) G\left(r_{i}, r_{j}\right) B_{i}\left(r_{i}\right) B_{j}\left(r_{j}\right) d Z_{2} d H_{2} d Y_{2} d X_{2} \\
\bar{R}_{i j} & =\int_{x_{i}} \int_{y_{i}} \int_{h_{i}} \int_{z_{i}} \frac{e^{-\frac{h_{i}^{2}}{2 \sigma^{2}}}}{\sqrt{2 \pi} \sigma} \rho B_{i}\left(r_{i}\right) B_{j}\left(r_{j}\right) d z_{i} d h_{i} d y_{i} d x_{i}
\end{aligned}
$$

The integration limits are defined by the boundaries of the corresponding wire segments.

\section{CORRECTION TERM FOR THE APPROXIMATION OF THE IMPEDANCE ENSEMBLE AVERAGE}

In [3] we have presented a method to compute the first order approximation of the average current distribution. In this section we demonstrate that using our stochastic high order basis function, we can efficiently calculate a correction term accurate up to third order for such approximation. Using Neumann expansion [7] the mesh current can be written as:

$$
I_{m} \simeq\left(I-{\overline{Z_{m}}}^{-1} \Delta+{\overline{Z_{m}}}^{-1} \Delta{\overline{Z_{m}}}^{-1} \Delta\right){\overline{Z_{m}}}^{-1} b
$$

where $\Delta=Z_{m}-\overline{Z_{m}}$, and $Z_{m}=M(R+j \omega L) M^{T}$. The first order approximation used in [3] for the average is $\overline{I_{m}}=E\left[I_{m}\right]={\overline{Z_{m}}}^{-1} b$. Since the average of the second order term is zero, when adding the correction term ${\overline{Z_{m}}}^{-1} \Delta{\overline{Z_{m}}}^{-1} \Delta{\overline{Z_{m}}}^{-1} b$ we can improve the approximation accuracy up to third order. The enhanced approximation of the average mesh current is therefore given by: ${\overline{I_{m}}}={\overline{Z_{m}}}^{-1} b+{\overline{Z_{m}}}^{-1} E\left[\Delta{\overline{Z_{m}}}^{-1} \Delta\right]{\overline{Z_{m}}}^{-1} b$. We calculate the correction term using a similar procedure as in [1], where the Kronecker product is used to write: $\operatorname{vec}\left(\Delta{\overline{Z_{m}}}^{-1} \Delta\right)=\left(\Delta^{T} \otimes \Delta\right) \overline{Z_{m}}$. The matrix $\Delta^{T} \otimes \Delta$ is of dimension $N^{2} \times N^{2}$, where $N$ is the number of basis functions. Every element of this matrix is determined by four wire segments and therefore the ensemble average of any such element is a function of four random variables:

$$
\begin{aligned}
& E[\Delta \otimes \Delta]_{i j}=\iiint \int_{X_{4}} \iiint \iiint \iint_{Y_{4}} \iiint \int_{Z_{4}} P_{4}\left(X_{4}, Y_{4}, Z_{4}, H_{4}\right) G\left(r_{1}, r_{2}\right) G\left(r_{3}, r_{4}\right) B_{1}\left(r_{1}\right) B_{2}\left(r_{2}\right) B_{3}\left(r_{3}\right) B_{4}\left(r_{4}\right) d Z_{4} d H_{4} d Y_{4} d X_{4}
\end{aligned}
$$

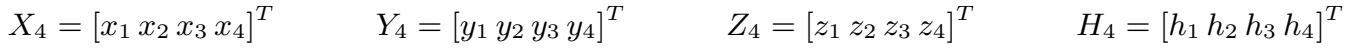

$$
\begin{aligned}
& P_{4}\left(X_{4}, Y_{4}, Z_{4}, H_{4}\right)=\frac{\exp \left(H_{4}{ }^{T} \Sigma_{4}^{-1} H_{4}\right)}{(2 \pi)^{2} \sqrt{\left|\Sigma_{4}\right|}}, \quad \Sigma_{4}=\sigma^{2}\left(\begin{array}{cccc}
1 & C\left(r_{1}, r_{2}\right) & C\left(r_{1}, r_{3}\right) & C\left(r_{1}, r_{4}\right) \\
C\left(r_{1}, r_{2}\right) & 1 & C\left(r_{2}, r_{3}\right) & C\left(r_{2}, r_{4}\right) \\
C\left(r_{1}, r_{3}\right) & C\left(r_{2}, r_{3}\right) & 1 & C\left(r_{3}, r_{4}\right) \\
C\left(r_{1}, r_{4}\right) & C\left(r_{2}, r_{4}\right) & C\left(r_{3}, r_{4}\right) & 1
\end{array}\right)
\end{aligned}
$$


where the integration limits are defined by the boundaries of the corresponding four segments. The integral in (8) is 16dimensional, and cannot be further reduced as it is not translational invariant. The size of the matrix required to store the elements of the matrix $\Delta^{T} \otimes \Delta$ grows with complexity $O\left(N^{4}\right)$, which, even for small size problems is practically untractable when using piecewise constant basis functions (e.g. $\mathrm{N}=1000$ ). On the contrary if we employ high order basis functions the same problems can be modeled with the same accuracy as shown in the result section by a system 20 times smaller $(\mathrm{N}=50)$, leading to time and memory requirements several orders of magnitude smaller. Finally, to further accelerate the calculation we propose an approximation of the integral in (8) by subdividing each wire segment along its length. The interaction between subsegments are calculated assuming the probability density function is space independent. The correlation distance between subsegments is approximated using the center to center distance. This assumption is valid provided the segmentation along the length is not too coarse.

$$
\begin{aligned}
E[\Delta \otimes \Delta]_{i j} \simeq & \sum_{\ell_{1}=1}^{N 1} \sum_{\ell_{2}=1}^{N 2} \sum_{\ell_{3}=1}^{N 3} \sum_{\ell_{4}=1}^{N 4} \iiint \int_{H_{4}} P_{4}\left(H_{4}, X_{c}^{\ell_{1}, \ell_{2}, \ell_{3}, \ell_{4}}, Y_{c}^{\ell_{1}, \ell_{2}, \ell_{3}, \ell_{4}}, Z_{c}^{\ell_{1}, \ell_{2}, \ell_{3}, \ell_{4}}\right) . \\
& \cdot \iiint \iiint \iiint \iiint G\left(r_{1}, r_{2}\right) G\left(r_{3}, r_{4}\right) B_{1}\left(r_{1}\right) B_{2}\left(r_{2}\right) B_{3}\left(r_{3}\right) B_{4}\left(r_{4}\right) d Z_{4} d Y_{4} d X_{4} d H_{4}, \\
= & \sum_{\ell_{4}}^{N 1} \sum_{\ell_{4}}^{N 2} \sum_{\ell_{2}=1}^{N 3} \sum_{\ell_{3}=1}^{N 4} \iiint \int_{\ell_{4}=1} P_{4}\left(H_{4}, X_{c}^{\ell_{1}, \ell_{2}, \ell_{3}, \ell_{4}}, Y_{c}^{\ell_{1}, \ell_{2}, \ell_{3}, \ell_{4}}, Z_{c}^{\ell_{1}, \ell_{2}, \ell_{3}, \ell_{4}}\right) d H_{4} . \\
& \cdot \iint_{X_{2}} \iint_{Y_{2}} \iint_{Z_{2}} G\left(r_{1}, r_{2}\right) B_{1}\left(r_{1}\right) B_{2}\left(r_{2}\right) d Z_{2} d Y_{2} d X_{2} \iiint_{X_{2}} \iiint_{Y_{2}} \iint_{Z_{2}} G\left(r_{3}, r_{4}\right) B_{3}\left(r_{3}\right) B_{4}\left(r_{4}\right) d Z_{2} d Y_{2} d X_{2},
\end{aligned}
$$

where $\ell_{i}$ is the index of the subsegments of wire segment $i$, and $X_{c}^{\ell_{1}, \ell_{2}, \ell_{3}, \ell_{4}}, Y_{c}^{\ell_{1}, \ell_{2}, \ell_{3}, \ell_{4}}$, and $Z_{c}^{\ell_{1}, \ell_{2}, \ell_{3}, \ell_{4}}$ contain the centers of the corresponding subsegments. Note that the probability density function $P_{4}$ is extracted out of the spatial integrals due to our space independency assumption. The inner integrals in (12) are translational invariant, and can be further reduced from dimension 6 to 3 using the technique presented in [3]. Consequently the total integral dimension is reduced from 16 to 7 , which can then be implemented in reasonable time.

\section{Results}

\section{A. Moderate size example}

To test the validity of our approach we used a relatively moderate example consisting of a square single turn inductor of outer diameter $20 \mathrm{~mm}$, and cross sectional dimensions of $0.5 \mathrm{~mm} \times 0.2 \mathrm{~mm}$. As a reference we used a fine cross-sectional discretization of $16 \times 8$ stochastic piecewise constant basis functions (SPCBF), which results in thin filaments of size smaller than a skin depth for the band of operation from $0.1 \mathrm{MHz}$ to $40 \mathrm{MHz}$. We then generated enough stochastic high order basis functions (SHOBF) so that the final error from the reference is less than $2 \%$ over the entire band of operation as demonstrated in Fig. 1. In Table I a detailed time analysis is presented to compare the performance of the SHOBF with that of the SPCBF. Despite the fact that the number of unique SHOBF integrals is $K^{2}$ less than the SPCBF integrals (K is defined in (4)), the matrix fill time did not reduce significantly. This is because one single SHOBF integral is exactly $K^{2}$ more expensive than one SPCBF integral (5). The reduction in the matrix solution time is however $O\left(R^{3}\right)$, where $R$ is the reduction in the number of basis functions, as a consequence of using gaussian elimination to solve the linear system. In this analysis the matrix vector product is not accelerated and no preconditioner is available, therefore iterative methods were not suitable at this time. It is observed that when used in the stochastic case, SHOBF provide a speed up even larger than in the deterministic case. We realize that the averaging operation smoothes the roughness impact on the current and that the SHOBF make use of this effect. This behavior cannot be utilized when using SPCBF and we need a very fine cross-sectional discretization.

TABLE I

DETAILED TIME ANALYSIS OF THE BASIS FUNCTION GENERATION AND COMPLETE COMPUTATION.

\begin{tabular}{|c||c||c||c|}
\hline Criterion & High order basis functions & Piecewise constant basis functions & Reduction ratio (R) \\
\hline Basis functions per cross-section & 6 & $8 \times 16$ & $>21.3$ \\
Basis function generation time & 2 minutes & - & - \\
Matrix fill time & 2 hours & 2 hours & 1 \\
Time for one solve & 2 seconds & 1 hour & 1800 \\
\hline
\end{tabular}

\section{B. Adding the correction term}

In Fig. 2 we demonstrate the effect of the correction term on the accuracy of our stochastic solver. The example is a single turn inductor of side length $20 \mathrm{~mm}$. The inductor is discretized in 32 segment. The cross sectional current per segment is approximated using $6 \mathrm{SHOBF}$, resulting in a total of $32 \times 6$ high order basis functions. The total time to calculate the 

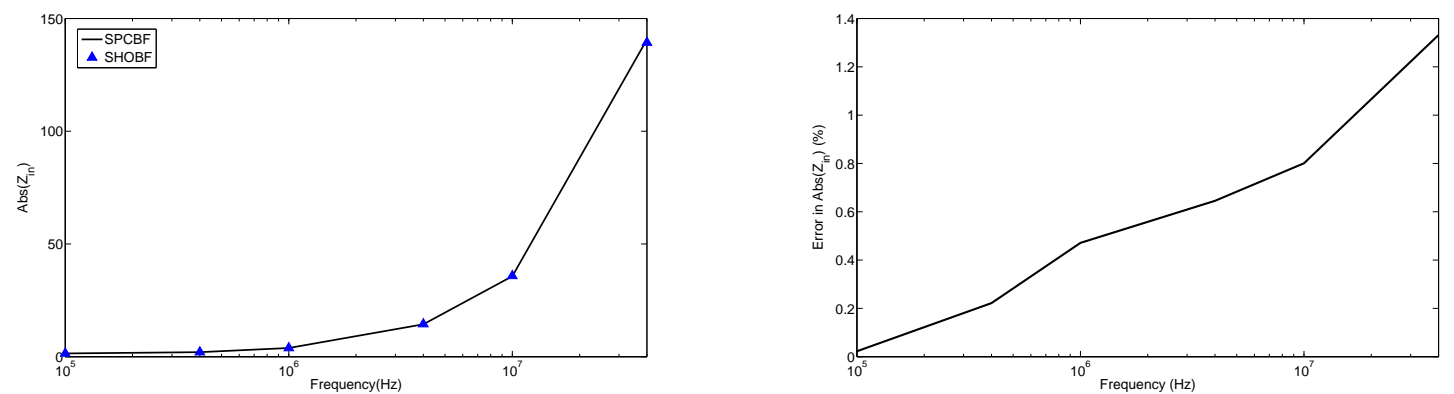

Fig. 1. Left: Magnitude of input impedance for SPCBF versus SHOBF. Right: Error in magnitude of impedance is less than $2 \%$ in the entire band of operation
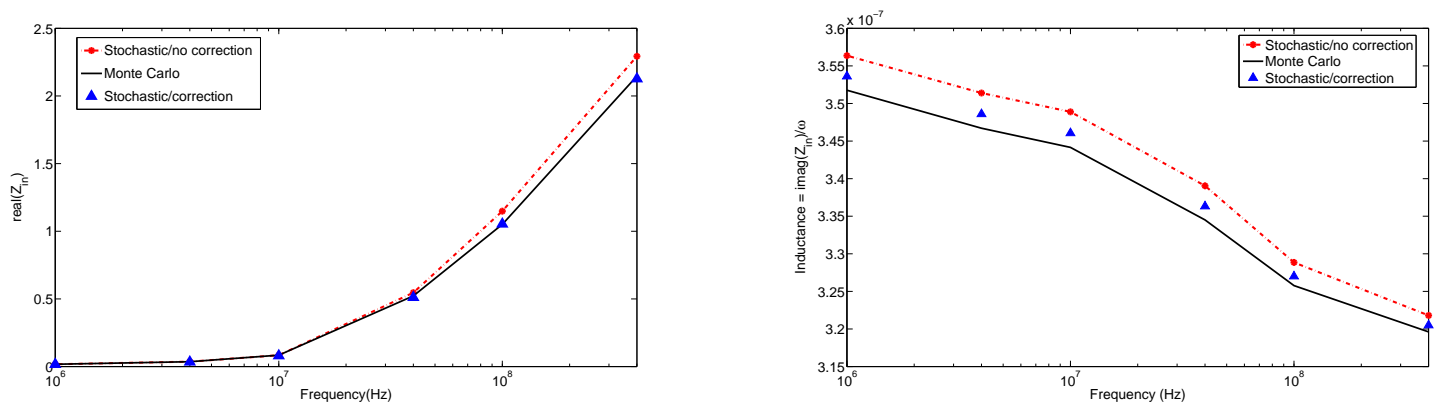

Fig. 2. Left: Effect of the correction term on the real part of average impedance . Right: Effect of the correction term on the average inductance

correction term is slightly less than 18 hours. The memory requirements to store the unique elements of the matrix $E[\Delta \otimes \Delta]$ is $1024 \times 1024$. If SPFBF were used for the discretization, 1024 basis functions would be needed to preserve the same accuracy, and the corresponding total storage requirement would be an unpractical matrix $1 M \times 1 M$. Finally, Fig. 2 clearly shows a significant reduction in the error between the first order approximation and the corrected one employing SHOBF, when compared to the reference Monte Carlo simulation.

\section{Large size example}

The next example is a large inductor structure that is practically impossible to solve in reasonable time and memory using SPCBF. This is a three turn inductor with an upper rough surface. We use 8 SHOBF per cross section to describe the current, resulting in a total number of unknowns: $32 \times 3 \times 8=768$. The matrix fill time is very large (about one day). The size of the matrix, if SPCBF were used, would be $12288 \times 12288$, which would be impossible to store and factorize using any standard personal computer. On the other hand the solve time for the SHOBF is less than one hour, which clearly proves the effectiveness of SHOBF in handling even previously untractable problems.

\section{CONCLUSION}

In this paper we have extended the high order basis functions to stochastic solvers. This extension is particularly necessary since the number of cross sectional filaments grows enormously in presence of rough surfaces. We have also utilized the high order basis functions to calculate a correction term necessary for the accurate calculation of the ensemble average of the impedance. The efficiency of the high order basis functions has been established on practical examples, and several order of magnitude reduction in both time and memory complexity have been shown.

\section{REFERENCES}

[1] Z. Zhu, A. Demir, J. White, "A stochastic Integral Equation Method for Modeling the Rough Surface Effect on Interconnect Capacitance" ICCAD 2004.

[2] H. Zhu, X. Zeng, W. Cai, J. Xue and D. Zhou, "A sparse grid based spectral stochastic collocation method for variations-aware capacitance extraction of interconnect under nanometer process technology", DATE 2007.

[3] T. Moselhy and L. Daniel, "Stochastic Volumetric MQS Formulation for Interconnect Extraction with Surface Roughness." submitted to EPEP 2007.

[4] L. Daniel, A. Sangiovanni-Vincentelli and J. White, "Proximity templates for modeling of skin and proximity effects on packages and high frequency interconnect.", ICCAD 2002.

[5] X. Hu, T. Moselhy, J. White and L. Daniel, "Optimization-based Wideband Basis Functions for Efficient Interconnect Extraction”, DATE, 2007.

[6] M. Kamon and M. J. Tsuk and J. White, "FASTHENRY: A Multipole-Accelerated 3-D Inductance Extraction Program" IEEE Trans. on Microwave Theory and Techniques, September 1994, vol. 42, no.9, p. 1750-1758.

[7] R. Ghanem and P. Spanos, Stochastic Finite Elements: A Spectral Approach, Spring-Verlag, 1991. 